\title{
Optimum Design of Thin Wideband Multilayer Electromagnetic Shield Using Evolutionary Algorithms
}

\author{
Kalyan S. Kola ${ }^{1}$, Debasis Mandal ${ }^{2 *}$, Jyotirmay Tewary ${ }^{3}$, \\ Ved P. Roy ${ }^{4}$, and Anup K. Bhattacharjee ${ }^{5}$ \\ ${ }^{1,2,3,4}$ Bengal College of Engineering and Technology, Durgapur, India \\ ${ }^{5}$ National Institute of Technology, Durgapur, India \\ *corresponding author, E-mail: deb.mandal22@gmail.com
}

\begin{abstract}
This paper describes the method of optimum design of multilayer perforated electromagnetic shield using evolutionary algorithms, namely Particle Swarm Optimization Algorithm (PSO) and Genetic Algorithm (GA). Different parameters which are inherently conflicting in nature corresponds to the multilayer structure of the electromagnetic shields have been considered. The goal is to minimize the overall mass of the shield with respect to its shielding effectiveness and cost. Three different models are considered and synthesized using evolutionary algorithms. Numerical optimal results for each model using different algorithms are presented and compared with each other to establish the effectiveness of the proposed method of designing.
\end{abstract}

\section{Introduction}

In this modern age of electronics Todays world, mostly depends and relies on the technology, which is based on electromagnetic waves and its radiations. This electromagnetic radiations have very adverse effects not only related to the human health issues causing various diseases, but it may also affect the performance of a device and degrades its efficiency. This phenomenon is known as electromagnetic interference. The only way of reduction or removal of such disturbances can be achieved by the means of electromagnetic shielding.

Electromagnetic shields have their properties to attenuate such unwanted radiation and capable to reduce and prevents coupling of such electromagnetic radiation to ensure electromagnetic compatibility. Thus the electromagnetic shields finds its wide range of applications in various electronic systems such as radar engineering, wireless communication, electromagnetic compatibility and much more. These are made engineers and researchers to concentrate more on the optimum design of electromagnetic shield considering various parameters which are inherently conflicting in nature [1-9]. Generally the goal of designing the shield is to obtain lightweight, cost effective electromagnetic model from a predefined database of existing materials shown in Table 1 which has the capabilities to attenuate electromagnetic radiations in a wide range of frequencies [2]. The shield may be in the form of single layer or multilayered structure. The single layer shield has their limitations of the narrow frequency band and thick structure therefore more concentration has been centered in the most recent years for the optimum designing of multilayer thin electromagnetic shields considering various parameters such as choice of materials, frequency range, number of layers, permeability, permittivity etc. Which has been solved successfully using different evolutionary algorithms $[10,11]$. This paper mainly focused on the optimum designing of thin wideband $(900-1800 \mathrm{MHz})$ perforated multilayer electromagnetic shield using Particle Swarm Optimization algorithm (PSO) $[12,13,18]$ and Genetic Algorithm (GA) [11,13-17]. During the problem formulation the total mass and cost of the shield, corresponding to the shielding effectiveness of the multilayer structure is considered to make the design practically more significant. Three different models are considered and compared to verify the effectiveness of the proposed design methods.

\section{Physical model of multilayer electromagnetic shield}

The basic model of the multilayer electromagnetic shield has been shown in Figure 1. The shield is characterized by its shielding effectiveness. The greater its value, the better is its shielding capabilities shown in Table 2. The shielding effectiveness can be defined as the ratio quantities at the receptor without the barrier and with the barrier.

$$
\begin{aligned}
& S E=20 \log _{10}\left(\frac{E_{0}}{E_{s}}\right) d B \quad \text { for E field } \\
& S E=20 \log _{10}\left(\frac{H_{0}}{H_{s}}\right) d B \quad \text { for } \mathrm{H} \text { field }
\end{aligned}
$$

Where $E_{0}$ and $H_{0}$ represent quantities at the receptor without shielding, Es and Hs denote quantities at the receptor with a shielding barrier between the emitter and the receptor. These expressions assume that the wave impedance is the same before and after the shield. In this article the optimum design of three different models of multilayer perforated electromagnetic shield formed by cascading of different materials having frequency dependent permitivities 


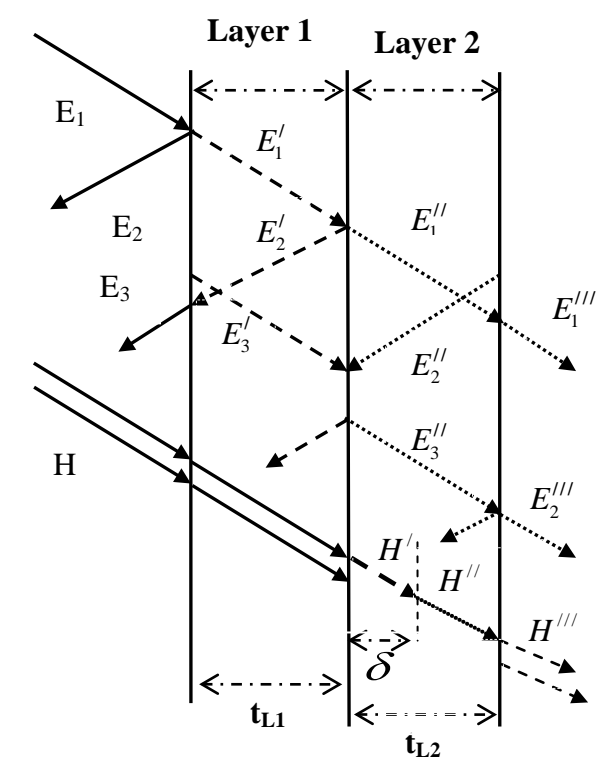

$\mathrm{E}=$ Electric Field, $\mathrm{H}=$ Magnetic Field, $\delta=$ skin depth, $\mathrm{t}=$ Thikness of Layer

Figure 1: Physical model of multilayer electromagnetic shield

and permiabilties using evolutionary algorithms have been proposed. The shielding effectiveness (SE) of such nonsolid (perforated) multilayer electromagnetic shield can be determined using the equations $(3-9)$.

$$
\begin{gathered}
S E=\left(A+R+B+K_{1}+K_{2}+K_{3}\right) d B \\
A=1.314 t \sqrt{f \mu \sigma} d B
\end{gathered}
$$

Where,

$A=$ Attenuation caused by the discontinuity,

$t=$ equivalent thickness of the multilayer shield,

$f=$ frequency of the radiated electromagnetic wave,

$\mu=$ equivalent relative permeability and

$\sigma=$ equivalent relative conductivity.

$R$ represents Loss due to single reflection $(\mathrm{dB})$.

$$
\begin{gathered}
R=168-10 \log _{10}\left(\frac{f \mu}{\sigma}\right) d B \\
B=20 \log _{10} e^{-\delta} d B
\end{gathered}
$$

$B=$ Multiple reflection loss $\delta=$ Skin depth.

$$
\delta=\frac{1}{\sqrt{\pi f \sigma}} d B
$$

$K_{1}=$ Correction term to account for the number of like discontinuities $(\mathrm{dB})$

$$
K_{1}=-10 \log _{10}(\text { an }) d B
$$

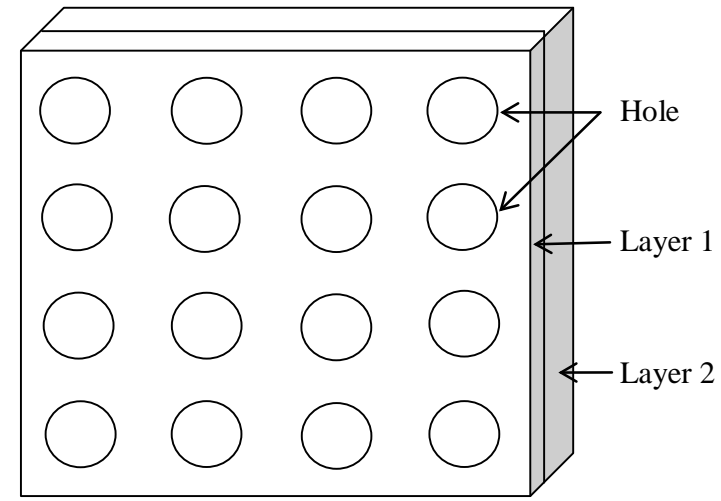

Figure 2: Cross section of multilayer perforated shield

Where, $a=$ Area of each hole $\left(\mathrm{cm}^{2}\right)$ and $n=$ Number of holes $/ \mathrm{cm}^{2}$.

$K_{2}=$ Low frequency correction term to account for the skin depth $(\mathrm{dB})$ which may be neglected at higher frequencies.

$K_{3}=$ Correction term to account for the coupling between the adjacent holes $(\mathrm{dB})$.

$$
K_{3}=0.20 \log _{10}\left\{\operatorname{coth}\left(\frac{A}{8.686}\right)\right\} d B
$$

Table 1: Database of shielding materials

\begin{tabular}{lcccc}
\hline Material & $\sigma_{r}$ & $\mu_{r}$ & $\sigma_{r} \mu_{r}$ & $\sigma_{r} / \mu_{r}$ \\
\hline Silver & 1.05 & 1 & 1.05 & 1.05 \\
Copper & 1 & 1 & 1 & 1 \\
Gold & 0.7 & 1 & 0.7 & 0.7 \\
Aluminum & 0.61 & 1 & 0.61 & 0.61 \\
Brass & 0.26 & 1 & 0.26 & 0.26 \\
Bronze & 0.18 & 1 & 0.18 & 0.18 \\
Tin & 0.15 & 1 & 0.15 & 0.15 \\
Lead & 0.08 & 1 & 0.08 & 0.08 \\
Nickel & 0.2 & 100 & 20 & $2 \times 10^{-3}$ \\
Stainless steel (430) & 0.02 & 500 & 10 & $4 \times 10^{-5}$ \\
Mu metal & 0.03 & $2 \times 10^{4}$ & 600 & $1.5 \times 10^{-6}$ \\
(at 1kHz) & & & & \\
Super permalloy & 0.03 & $10^{5}$ & 3000 & $3 \times 10^{-7}$ \\
(at 1kHz) & & & & \\
Permalloy & 0.027 & $2 \times 10^{4}$ & 540 & $1.35 \times 10^{-6}$ \\
\hline
\end{tabular}

\section{Problem Formulation}

The shield should capable to minimize the effects of electromagnetic radiation by providing significant shielding effectiveness (SE) having practically possible least effective mass and thickness with low cost is the primary objective of our design, therefore all these design considerations are incorporated in the form of the following objective function 
for the purpose of optimization.

$$
Z / c m^{2}=(1-a n)\left(t_{L 1} \rho_{L 1}+t_{L 2} \rho_{L 2}\right)
$$

Subject to $S E \geqslant 50$

Where, $54.5415 \leqslant a \leqslant 218.1662,0<n \leqslant 1$

$t_{L 1}=$ Thickness of layer 1 ,

$\rho_{L 1}=$ density of material (layer 1 ),

$t_{L 2}=$ Thickness of layer 2 ,

$\rho_{L 2}=$ density of material (layer 2 ).

Thickness ranges (in $\mathrm{mm}$ ) for different materials used in shielding models are chosen as follows.

Model 1: $0.1 \leqslant t_{c u} \leqslant 0.5$ and $0.005 \leqslant t_{\text {per }} \leqslant 0.1$

Model 2: $0.1 \leqslant t_{\text {steel }} \leqslant 3$ and $0.005 \leqslant t_{\text {per }} \leqslant 0.1$

Model 3: $0.1 \leqslant t_{\text {Tin }} \leqslant 0.5$ and $0.005 \leqslant t_{\text {per }} \leqslant 0.1$

Table 2: Some standard values of shielding effectiveness

\begin{tabular}{lcc}
\hline SE $(\mathrm{dB})$ & Attenuation ratio & Results \\
\hline 20 & $10: 1$ & Normal acceptable shielding \\
40 & $100: 1$ & Normal acceptable shielding \\
60 & $1000: 1$ & Normal acceptable shielding \\
80 & $10000: 1$ & Normal acceptable shielding \\
100 & $100000: 1$ & Above average shielding \\
120 & $10^{6}: 1$ & Above average shielding \\
\hline
\end{tabular}

\section{Algorithm Overviews (PSO and GA)}

The basic concept of PSO [12,13,18] algorithm which is based on the swarm behavior of insects, animals herding, birds flocking and fish schooling was first developed and introduced by Kennedy and Eberhart in 1995 where the search pattern of each swarm is adapted from its own as well as other members experience. In PSO every individual member of the swarm is called particle and it represents the potential solution which is a point in the search space and the global optimum is the location of the food. The fitness value and a velocity corresponding to the best experiences of the swarm to search for the Global optimum in the Ddimensional search space of each particle adjust its flying direction. Moreover the PSO algorithm is easy to implement and has been empirically shown to perform well on many optimization problems.

Whereas Genetic Algorithm (GA) [11,13-17] is a nature inspired evolutionary algorithm based on individual population, which is characterized by its fitness function. Three main steps involved in GA are Selection, Crossover and Mutation to generate the new solution. The higher fitness value provides better results and accordingly parents are selected to reproduce offspring for a new generation with more chance to reproduce for the individual having higher fitness value. Both the new and old generation having the same population size and old generation dies. New offspring having the properties of both new as well as old generation. Finally, the population will converge to the optimal solution.
Table 3: Parametric setup of PSO and GA algorithm

\begin{tabular}{lc}
\hline Parameters & Values \\
\hline Swarm size & 50 \\
Initial population & Random \\
Cc1 1.4945 \\
C2 $=1.4945$ \\
Inertia Weight & Damp linearly from 0.9 \\
to 0.2 with iterations \\
VRmin & 0 \\
VRmax & 1 \\
Run no & 20 \\
Termination condition & 1000 iterations (max) \\
& \\
\hline & GA \\
Parameters & Values \\
\hline Population size & 50 \\
Crossover (CR) & 2 \\
Crossover probability & 0.08 \\
Mutation Probability & 0.02 \\
Selection & Roulette Wheel \\
Run no & 20 \\
Termination condition & 1000 iterations (max) \\
\hline
\end{tabular}

\section{Results and Discussion}

Three different models of multilayer wideband $(900 \mathrm{MHz}-$ $1800 \mathrm{MHz}$ ) electromagnetic shield are designed and synthesize. For model1 copper and permalloy, for model 2 steel and permalloy and for model 3 tin and permalloy has been considered. Numerical optimal results for each model applying different evolutionary algorithms are presented and compared with others to establish the effectiveness of the proposed design method in Table 4 and Table 5.

Table 4. shows thickness of individual materials used for designing the different models of electromagnetic shield along with the area of each hole and number of holes per $\mathrm{cm}^{2}$ as well, obtained using PSO and GA. Considering $1000 \mathrm{~cm}^{2}$ areas to be shielded, Table 5 describes that for model1 (Copper and Permalloy) PSO consequences shield with lower mass which exhibit better shielding effectiveness over the desired frequency band as compare to GA. Similarly for model 2 (Steel and Permalloy) it has been found that though both PSO and GA depicts the same results as far as the mass of the shield is concerned, but PSO provides the better shielding effectiveness as compared to GA over the desired frequency band. Lastly model 3 (Tin and Permalloy) is presented where it has been observed that PSO results in shield with lower mass than GA, whereas GA provides slightly better shielding effectiveness than PSO in the desired range of frequency. Thus, it has also been observed that regarding the designing of the shield PSO comes out as a clear winner over GA for this design aspect. 
Table 4: Parameters for electromagnetic shield design for frequency band (900-1800 MHz) using Evolutionary Algorithms

\begin{tabular}{lccccccc}
\hline Model & Material used & \multicolumn{2}{c}{$\begin{array}{c}\text { Thickness } \\
(\mathrm{mm})\end{array}$} & \multicolumn{2}{c}{$\begin{array}{c}\text { Area of each hole } \\
\left(\mathrm{cm}^{2}\right)\end{array}$} & \multicolumn{2}{c}{ Number of holes/ $\mathrm{cm}^{2}$} \\
& & PSO & GA & PSO & GA & PSO & GA \\
\hline \multirow{2}{*}{1} & $\begin{array}{c}\text { Copper } \\
\text { Permalloy }\end{array}$ & 0.1329 & 0.1019 & 132.5792 & 217.538 & 0.0071 & 0.0031 \\
& & 0.0052 & & & & & \\
2 & Steel & 0.1367 & 0.100 & 103.5472 & 71.5228 & 0.0091 & 0.0128 \\
& Permalloy & 0.0409 & 0.0363 & & & & \\
3 & Tin & 0.1457 & 0.1347 & 120.6567 & 66.4165 & 0.0078 & 0.0138 \\
\hline
\end{tabular}

Table 5: Mass and Shielding effectiveness of the electromagnetic shield

\begin{tabular}{lcccccccc}
\hline Model & Material used & \multicolumn{2}{c}{$\begin{array}{c}\text { Effective mass } \\
\left(\mathrm{gm} / \mathrm{cm}^{2}\right)\end{array}$} & $\begin{array}{c}\text { Area to be shielded } \\
\left(\mathrm{cm}^{2}\right)\end{array}$ & \multicolumn{2}{c}{$\begin{array}{c}\text { Mass of the shield } \\
(\mathrm{gm})\end{array}$} & \multicolumn{2}{c}{$\begin{array}{c}\text { Shielding Effectiveness (SE) } \\
(\mathrm{dB})\end{array}$} \\
\hline 1 & $\begin{array}{c}\text { PSO } \\
\text { Copper }\end{array}$ & GA & & GA & PSO & GA \\
\hline & $\begin{array}{c}\text { Permalloy } \\
\text { Steel }\end{array}$ & 0.1007 & 0.3078 & & 100.7 & 307.8 & 98.0449 & 92.9386 \\
& $\begin{array}{c}\text { Permalloy } \\
3\end{array}$ & 0.1000 & 0.1000 & 1000 & 100 & 100 & 75.0624 & 73.8164 \\
\hline
\end{tabular}

For carrying out the simulation all the algorithms are run for 20 independent trials with maximum number of iterations is set to 1000 in each time. Computations have been done in MATLAB 2010a with core 2 duo processor $3 \mathrm{GHz}$ with $2 \mathrm{~GB}$ RAM.

\section{Conclusions}

An optimized model of multilayer thin wideband (900 $\mathrm{MHz}-1800 \mathrm{MHz}$ ) perforated electromagnetic shield has been proposed and assessed. Three different models are designed and analyzed using evolutionary algorithms (PSO and GA). It has been observed that all the three models are capable to provide shielding effectiveness which is more than the 'normal acceptable shielding range' $(\geq 50 \mathrm{~dB})$, hence, the models can be chosen or preferred based on the requirement for its different practical aspects subject to the Shielding effectiveness, mass and cost of the shield.

Simulated results for different models clearly established this fact and the effectiveness of the proposed method as well. The method is also applicable for other combination of available shielding materials.

\section{References}

[1] Hemming, L. H., Architectural Electromagnetic Shielding Hand book: A Design and Specification Guide, New York, USA: IEEE Press, 1992.
[2] Chung, D. D. L, Materials for Electromagnetic Interference Shielding, Journal of Materials Engineering and performance, Vol. 9(3), 350-354, June 2000.

[3] Kieser, Bernhard, Principle of Electromagnetic compatibility, Artech House, 1987.

[4] Saliovich, A. B., Electromagnetic Shielding Handbook for Wired and Wireless Emc Applications, Kluwer Academic Pub, 1999.

[5] Kaiser, K. L., Electromagnetic Shielding, Taylor \& Francis, 2005

[6] Mottahed, B. D. and Manoochehri, S, Joint design methodology based on the electromagnetic shielding effectiveness capabilities, IEEE Transactions on Components, Packaging, and Manufacturing Technology: Part B, Vol. 19,No. 1, 238-247, Feb 1996.

[7] Colotti, J., EMC Design Fundamentals, Systems, Applications and Technology Conference, 2006. LISAT 2006. IEEE Long Island, NY, pp. 1-2, 2006.

[8] Schulz R. B., Plantz, V. C. and Brush, D. R., Shielding theory and practice, IEEE Transactions on Electromagnetic Compatibility, Vol. 30, No. 3, 187-201, Aug 1988. 
[9] Biswas, S., Rakshit, P., Dey, T. K., Design of wire mesh shield-an analytical approach and testing of its performance, Electro Magnetic Interference and Compatibility (INCEMIC), 2006 Proceedings of the 9th International Conference on, Bangalore, 132137, 2006.

[10] Antonini, G. and Orlandi, A., Optimal electromagnetic shielding design considering multiple constraints, Electromagnetic Compatibility, 2000. IEEE International Symposium, Vol. 2, 711-716, Washington DC, 2000.

[11] Ziolkowski, M. and Gratkowski, S. R., Genetic Algorithm and Bezier Curves-Based Shape Optimization of Conducting Shields for Low-Frequency Magnetic Fields,IEEE Transactions on Magnetics, Vol. 44, No. 06, June 2008.

[12] Kennedy, J. and Eberhart, R. C., Particle swarm optimization. Proceedings of IEEE International Conference on Neural Networks, Piscataway, NJ., 19421948, 1995

[13] Boeringer, D. W. and Werner, D. H., Particle swarm optimization versus genetic algorithms for phased array synthesis, IEEE Trans. Antennas Propagat., Vol. 52, 771-779, 2004.
[14] Haupt, R. L., Introduction to genetic algorithms for electromagnetics, IEEE Antennas and Propagation Magazine, Vol. 37, No. 2, 7-15, 1995.

[15] Jain, R. and Mani, G. S., Solving Antenna Array Thinning Problem Using Genetic Algorithm, Applied Computational Intelligence and Soft Computing, Vol. 2012, pp.14, 2012.

[16] Man, K., Tang, K. and Kwong, S., Genetic algorithms: concepts and applications [in engineering design], Industrial Electronics, IEEE Transactions on, vol. 43, no. 5, pp. 519-534, Oct 1996.

[17] Marcano, D. and Duran, F., Synthesis of antenna arrays using genetic algorithms, Antennas and Propagation Magazine, IEEE, vol. 42, no. 3, pp. 12-20, Jun 2000.

[18] Mandal, D., Kola, K. S. and Bhattacharjee, A., Sidelobe Reduction of a Scanned Circular Array using Particle Swarm Optimization Algorithm,International Journal of Recent Trends in Electrical and Electronics Engg.,vol. 3, no. 1, pp. 54-61, Dec. 2013. 